\title{
BSE fostered by cosinesss and lack of independent advice
}

\section{Japan needs experienced scientists in positions of power to make reliable assessments of government advice}

Sir - Your Opinion article "Japan's beef scandal" (Nature 413, 333; 2001) had an extraordinary impact on the Japanese mass media. Many newspapers and television news shows criticized the government's irresponsible attitude to bovine spongiform encephalopathy (BSE, or madcow disease) by citing your article.

You pointed out the cosy relationships between government and industry and between government and healthcare institutions, strengthened by the former ministry officials, known as amakudari (because they have 'descended from heaven'), who retired from government to join large companies. This is a major reason why the government did not take immediate action to prevent the spread of this dreadful disease. It seems to me that official comments about a "lack of scientific evidence" for the risk of BSE development results from a lack of scientists in the government.

Although many scientists with PhDs are now working for the government, most of them are in scientific research institutes belonging to the ministries. Only a few scientists with research experience are high-ranking officials. Political decisions related to science and health are made by recommendations from government advisory councils. Because high-ranking officials cannot understand the scientific logic behind this advice, they tend to accept it without criticism or question. Therefore, political decisions are almost completely dependent on the people appointed to these advisory boards. Cosy relationships between government and industry are fostered when officials select members of advisory councils.

I believe that Japan should make a prompt effort to increase the number of scientists with appropriate research experience in the various ministries, especially those for health and science. Good scientists can well understand from their experience how trivial problems can lead to a fatal catastrophe. In addition, they have been trained to make judgements based on scientific logic, rather than to accept assertions uncritically.

I think that Japanese scientists and scientific societies should also feel some responsibility for the present situation. They have never spoken out or made efforts to increase the number of scientists in government. Nowadays, our daily lives cannot be detached from science. Japanese scientists should understand the present requirements of our nation, and take the initiative to change an irresponsible system. Mitsuo Tagaya

School of Life Science, Tokyo University of Pharmacy and Life Science, Horinouchi, Hachioji 192-0392, Japan
Meat and bone meal still used in animal feed

Sir — In your News Story, “Japan's first BSE case fuels fears elsewhere" (Nature 413, 337; 2001), you state that "British farmers dumped [cattle feed containing animal parts] in the region [East Asia] when demand slumped in Europe in the early 1990s". This is a careless statement, not up to the normal standards of your journal. British farmers did not and do not export animal feed. It is feed manufacturers who export feed.

As made clear in the exhaustive BSE inquiry report (see www.bseinquiry.gov. uk), during 1989 and 1990 the UK authorities informed the Office International des Epizooties and the veterinary authorities in all countries importing meat and bone meal from the United Kingdom of the feed ban on UK domestic ruminants.
The use of meat and bone meal in feed for non-ruminants (pigs and poultry) was legal in the United Kingdom until August 1996, and until January 2001 in the rest of the European Union (EU).

Meat and bone meal is still being used in animal feed in many non-EU countries, despite the BSE experiences of the United Kngdom and of the rest of the EU.

Stephen Rossides

Food, Health and Science Department, National Farmers' Union, 164 Shaftesbury Avenue, London WC2H $8 H L, U K$

\section{Tantalizing glimpse of a vanishing dinosaur}

Sir - Your News item "Elusive fossil could conceal answer to dinosaur debate" (Nature 412, 844, 2001) about the Chinese psittacosaurid with preserved integuments prompts me to add some information. Early last year, I was invited to travel to the Museo Civico di Storia Naturale in Milan, and hence was able to examine the specimen in some detail. I was satisfied that the integumentary remains are real, and apparently different from the feathers or protofeathers seen on some theropod specimens from the same provenance.

At the time, my Italian colleagues in Milan hoped that a joint study of the specimen with Chinese colleagues could be arranged, after which the fossil would be returned to China. Together with my wife, Dr Haiyan Tong, and others, we tried to have the psittacosaur returned to a Chinese institution, by providing possible contacts in China. But our attempt failed and the specimen was removed from the Milan museum.

I do not know the exact reason for our failure, but one result was that plans for a joint study in Milan were dropped. I am ignorant of the present whereabouts of the specimen. Of course, I have always believed that the fossil should be returned to its country of origin, and I have no intention of publishing anything formal about it, other than this brief note, which I hope may shed some light.

Eric Buffetaut

Centre National de la Recherche Scientifique, 16 cour du Liégat, 75013 Paris, France

\section{Once again, insects worked it out first}

Sir-Because of the contact between phases, transport of liquid droplets on solid surfaces is a complicated, nonefficient task requiring a considerable amount of energy. Increasing the contact angle between the liquid and solid (hydrophobicity) above $120^{\circ}$ should ease the motion. By coating a small amount of liquid with grain spores of Lycopodium, Aussillous and Quéré ${ }^{1}$ report their creation of non-sticky liquid marbles (contact angle of $\left.180^{\circ}\right)$. The droplets are soft solid and elastic, and can bounce and roll on a solid surface without leaking. It was predicted in the News and Views article ${ }^{2}$ accompanying this paper that this brilliant trick will have a rapid practical use in wear-free micromachines and lubrication.

Yet tiny, relatively primitive insects faced and solved a similar problem millions of years ago. Homopterans (aphids, whiteflies, and so on) feed on plant sap (usually phloem) that contains a high concentration of sugars but low nitrogen levels. Therefore, the extra sugars are deposited by the insects as sticky droplets, commonly known as honeydew. Some aphids live in galls where they face 
the danger of contamination and drowning in their own honeydew. These insects coat the honeydew with wax secreted from specialized glands ${ }^{3}$.

Furthermore, some gall-forming aphid species clean their galls by pushing the coated honeydew outside ${ }^{4-6}$. The coated elastic honeydew droplets can be pushed, rolled and squeezed without leaking or wetting. $\mathrm{Smith}^{3}$ noted that this behaviour was initially observed by Buckton in 1876 and may have promoted the evolution of social organization in aphids ${ }^{7}$. Practically, it is worthwhile examining whether aphid's wax is better at coating liquid droplets than are the spores of Lycopodium.

In short, 'liquid marbles' are yet another example of how insects 'developed' a technology through natural selection long before humans got around to it ${ }^{6}$. Technologists (high and low) and engineers should look for new solutions with the eyes of biologists.

M. Inbar ${ }^{\star}$, J. C. Schultz $\dagger$

${ }^{\star}$ Department of Biology, University of Haifa at Oranim, Tivon 36006, Israel

$\dagger$ Department of Entomology, Pennsylvania

State University, University Park,

Pennsylvannia 16802, USA

1. Aussillous, P. \& Quere, D. Nature 411, 924-927 (2001).

2. Mahadevan, L. Nature 411, 895-896 (2001).

3. Smith, R. G. Journal of Natural History 33, 513-530 (1999).

4. Aoki, S. Kontyû 48, 71-73 (1980).

5. Kurosu, U. \& Aoki, S. Journal of Ethology 9, 51-55 (1991).

6. Benton, T. G. \& Foster, W. A. Proceedings of the Royal Society of London 247, 199-202 (1992).

7. Stern, D. L. \& Foster, W. A. Biological Review 71, 27-79 (1996)

8. Akre, R. D. et al. Insects Did it First (Ye Galleon Press,

Washington, 1992).

\section{Disclosure of interests: there's a long way to go}

Sir - I read with great interest and approval your policy (Nature 412, 751; 2001) requiring authors to disclose financial interests. This policy has been sorely needed for a long time, and I hope that other journals follow suit. My only caveat is with respect to your anticipation that "employers" will "police" the policy.

I recently attended a symposium chaired by two key university counsel from two leading US universities (one private, one state), and asked them what they and their institutions do to ensure that scientists disclose financial interests in publishing articles. The answer was a blank stare - they assumed that such interests are disclosed as appropriate and have no procedures or policies for ensuring that such disclosures occur.

Tamsen Valoir

Jenkens \& Gilchrist, 1100 Louisiana Street, Suite

1800, Houston, Texas 77002, USA

\section{Sequenced strains must be saved from extinction}

Sir - Many prokaryotic strains used for genome sequencing projects are poorly documented and not generally available.

With improvements in sequencing technology and growing recognition of the value of microbial genome sequence data, the number of microbial genomesequencing projects is increasing rapidly. There are 56 completed prokaryotic genome sequences (10 strains of Archaea and 46 of Bacteria), and another 210 in progress (see, for example, www.tigr.org and www.integratedgenomics.com).

Of these 266 projects, some of which are performed on organisms not available in pure culture - some endosymbionts, for example - only 51 represent the type strain of the species. (A type strain is made up of living cultures of an organism descended from the nomenclatural type.) Of the rest, 138 represent non-type strains (a non-type strain is often selected only because it happens to be close at hand); 31 projects concern symbionts and environmental (uncultured) strains; 32 do not specify a strain; 14 represent prokaryotic species with invalid species names (validly named bacterial species are either on the 1980 approved lists of names, or validated after 1980 by taxonomic description in the International Journal of Systematic and Evolutionary Microbiology, or by validation in that journal: an invalid name has no standing in nomenclature and may be changed subsequently. Only 115 represent the type species of the genus (the typus of the genus included when the genus name was originally validly published) and only 123 are deposited in public culture collections. Mandatory deposition of the type strain of any validly described (culturable) prokaryotic species in a major public culture collection guarantees the availability of the strain and allows crossreferencing of published data.

When there were only a few projects, these taxonomic and preservation issues were not so evident. With the explosion in sequencing and the sequencing of multiple strains of a species (including Escherichia coli, Staphylococcus aureus and other major pathogens), questions of strain identity and safekeeping assume more importance. Deposition of strains in public collections with long-term funding is the only way to ensure their maintenance and their continuing availability to the scientific community. As things stand, it is a real possibility that a strain for which a wealth of genomic data has been generated may become "extinct" through loss of viability.

We propose that the following standards should be adopted by the entire community. First, genome-sequencing project lists and databases should include the name of the strain sequenced and its associated culture collection accession number(s), as well as its origin. Second, the type strain of a species should be used for sequencing unless other factors make this inappropriate. Third, strains for which genome sequences have been, or are being, generated should be deposited in at least two major public biological resource centres, such as the American Type Culture Collection, the German Collection of Microorganisms and Cell Cultures, the Pasteur Institute Collection or the Japanese Collection of Microorganisms. Naomi Ward ${ }^{\star}$, Jonathan Eisen ${ }^{\star}$, Claire Fraser ${ }^{\star}$, Erko Stackebrandt $\dagger$

${ }^{*}$ The Institute for Genomic Research, 9712 Medical Center Drive, Rockville, Maryland 20850, USA † German Collection of Microorganisms and Cell Cultures, Mascheroder Weg 1b, D-38124 Braunschweig, Germany

\section{Taxonomists make a name for themselves}

Sir - Many publications by taxonomists are not included in the Science Citation Index $(S C I)$, as pointed out in

Correspondence by A. G. Valdecasas et al. (Nature 403, 698; 2000) and by F. T. Krell (Nature 405, 507-508, 2000). E. Garfield (Nature 413, 107; 2001), on the other hand, pointed out some citation classics in taxonomy based on the SCI.

Your correspondents fail to mention the real reason most taxonomists are not included in the SCI: the way taxonomic research is cited means it does not feature in references. In referring to the name of a species, it is customary to state the name(s) of the author(s) after the species name, sometimes abbreviated and with the year of publication of the first description of the species. This citation method is ignored by the SCI and other citation indexes, which is why these scientists do not get the credit they deserve, even though each use of a species name in fact represents a citation.

A quick look in the ISI Web of Science 1988-2001 [www.isinet.com/isi/products/ citation/wos/index.html] shows that the most frequently cited scientists are the authors who named the bacterium Escherichia coli (108,262 citations), the yeast Saccharomyces cerevisiae $(43,403)$ and the fruitfly Drosophila melanogaster $(14,451)$. To put these numbers into perspective, Albert Einstein is cited 'only' 11,920 times.

\section{Gerard van der Velde}

Department of Animal Ecology and Ecophysiology, University of Nijmegen, Nijmegen, Toernooiveld 1, 6525 ED, The Netherlands 